\title{
Dissociation Mechanics and Stability of Type a Botulinum Neurotoxin Complex by Means of Biophysical Evaluation
}

\section{Shavron Hada}

Dongguk University

Jae Chul Lee

Dongguk University

\section{Eun Chae Lee}

Dongguk University

\section{Sunkyong Ji}

Dongguk University

\section{Jeong Sun Nam}

Jetema

Bum Jin Yun

Jetema

\section{Dong Hee $\mathrm{Na}$}

Chung-Ang University

Nam Ah Kim

Dongguk University

Seong Hoon Jeong ( $\square$ shjeong@dongguk.edu )

Dongguk University

\section{Research Article}

Keywords: Botulinum neurotoxin A complex, Protein aggregation, Biophysical characterization, Sizeexclusion chromatography (SEC), Formulation, Neurotoxin stability

Posted Date: January 6th, 2022

DOI: https://doi.org/10.21203/rs.3.rs-1224293/v1

License: (c) (i) This work is licensed under a Creative Commons Attribution 4.0 International License.

Read Full License 
Version of Record: A version of this preprint was published at Journal of Pharmaceutical Investigation on April 8th, 2022. See the published version at https://doi.org/10.1007/s40005-022-00570-2. 


\section{Abstract}

Biophysical characterization of type A botulinum neurotoxin (BoNT/A) complex along with its thermodynamic stability was assessed through a combination of various methods. BoNT/A exists as large complexes in association with neurotoxin associated proteins (NAPs). To evaluate its biophysical behavior, size-exclusion chromatography (SEC), multi-angled light scattering (MALS), enzyme linked immunosorbent assay (ELISA), and dynamic light scattering (DLS) were utilized. Initially, a single peak (peak 1) of SEC was observed at pH 6.0, and an additional peak (peak 2) appeared at pH 7.4 with a decrement of peak 1. Through MALS and ELISA, the peak 2 was determined to be BoNT/A dissociated from its complex. The dissociation was accelerated by time and temperature. At $37^{\circ} \mathrm{C}$, dissociated BoNT/A self-associated at $\mathrm{pH} 7.4$ in the presence of polysorbate 20. On the other hand, the dissociation was partly reversible when titrated back to $\mathrm{pH}$ 6.0. Overall, BoNT/A was more stable when associated with NAPs at pH 6.0 compared to its dissociated state at $\mathrm{pH}$ 7.4. The conventional analytical methods could be utilized to relatively quantify its amount in different formulations.

\section{Introduction}

Type A botulinum neurotoxin (BoNT/A), a $150 \mathrm{kDa}$ protein, is comprised of a $50 \mathrm{kDa}$ light chain and a $100 \mathrm{kDa}$ heavy chain, covalently linked by an inter-chain disulfide bond ${ }^{1,2}$. It is generally produced in association with a group of neurotoxin-associated proteins (NAPs), comprising a non-toxic nonhemagglutinating subunit (NTNHA) and hemagglutinin subunits (HAs) HA-17, HA-33, and HA-70, yielding the final molecular weight of $\sim 900 \mathrm{kDa}^{3}$. The majority of botulinum neurotoxin products available on the market are in the form of purified neurotoxin complexes, except for Xeomin ${ }^{\circledR}$, which includes a $150 \mathrm{kDa}$ neurotoxin without the complexing proteins ${ }^{4}$. Meanwhile, as reported earlier, NAP can enhance the structural stability and activity of the neurotoxin (e.g., BoNT/A) ${ }^{5-8}$. Its stabilizing effect can even maintain its oral toxicity ${ }^{9-12}$. Nevertheless, the pharmacological effect is dependent on the neurotoxin dissociated from the complex 2,13,14.

Botulinum neurotoxin products such as Botox ${ }^{\circledR}$, Dysport ${ }^{\circledR}$ and Xeomin $\AA$ are marketed as vacuum- or freeze-dried powder set at $\mathrm{pH}$ around 7, whereas Myobloc ${ }^{\circledR}$ is a solution for injection set at pH around 5.6 15,16 . Botox $\AA$, Dysport ${ }^{\circledR}$ and Xeomin $\AA$ are commercially available botulinum toxin type $A$, and Myobloc $\AA$ is type $B$, suggesting the conformational and colloidal stability of each toxin could differ with its types. Supportively, the conformational stability of therapeutic proteins and antibodies are optimal in very narrow $\mathrm{pH}$ range and their unique balances of protein-protein interactions induce different colloidal stability ${ }^{17-20}$. Besides, earlier studies suggested that the $\mathrm{pH}$ and ionic strength can be major driving forces for the dissociation of neurotoxin from its complex ${ }^{13,14,21}$. Neurotoxins can readily dissociate from the complex within a minute, especially when exposed to alkaline $\mathrm{pH}$, suggesting the existence of a free form (i.e., dissociated) of botulinum neurotoxin as reconstituted ${ }^{22,23}$. At acidic $\mathrm{pH}$, the protective activity of NAPs on the neurotoxin indicates they maintain their associated state with the neurotoxin $7,24,25$ 
On the other hand, all products mentioned contain human serum albumin (HSA) in the formulation to protect BoNT/A from nonspecific binding ${ }^{26}$. It stabilizes the neurotoxic proteins during and after manufacturing, suppressing the aggregation of neurotoxic proteins ${ }^{27}$. However, the inclusion of HSA introduces the risk of viral and other pathogens transmission since it is derived from humans along with the difficulty in maintaining a uniform quality ${ }^{28}$. To counter this risk, recombinant HSA could be an alternative. However, challenges still exist in the analytical method development, as the selective analysis and quantification of the active proteins and their degradation products or aggregates are often difficult in presence of the secondary protein in the form of HSA. For the reasons, the market demands HSA-free formulations of botulinum toxins. Consequently, the stability of the toxins and the mechanism of its dissociation should be investigated thoroughly.

In fact, the existence of the neurotoxin as a complex with NAPs poses challenges, as each of the proteins in the complex portrays individual behavior in different solution conditions. Given their complexity, different methods intended to investigate their similar attributes are often necessary to provide independent confirmation of the protein properties. Enzyme-linked immunoassay (ELISA) has been widely used as a highly sensitive quantification tool for neurotoxins ${ }^{29-32}$. However, the process is limited to detection and quantification of neurotoxin regardless of its associated or dissociated state as the neurotoxins are exposed to reagents at $\mathrm{pH} 7.2$ to 7.4 during the analysis.

For size exclusion chromatography (SEC), there is no relevant report of its use in quantifying and discriminating associated and dissociated neurotoxin. Generally, this analysis has been conventionally used for the quantification of proteins in various states as monomers, oligomers, aggregates, and even fragments. The major benefit is the use of elution conditions that allow the characterization of proteins with the least impact in their local environment and conformational structure ${ }^{33}$. In this study, the state of neurotoxin was investigated mainly using SEC to evaluate the dissociated BoNT/A from NAPs at different pHs, incubation times, and storage temperatures. Moreover, the impact of polysorbate 20 is investigated since it has been used as a stabilizer in marketed formulations and can be an excipient as a substitute for HSA ${ }^{28,34,35}$. Additionally, multiangle light scattering (MALS) and ELISA were utilized further to interpret the SEC chromatograms. Lastly, dynamic light scattering (DLS) was used to access the size distribution and zeta potential of the complexes at different $\mathrm{pHs}$.

\section{Experimental Section}

\subsection{Materials and sample preparation}

Type A botulinum neurotoxin complex (with an approximate molecular weight of $900 \mathrm{kDa}$ ) was supplied by JETEMA Co., Ltd. (Seoul, South Korea) as a $1.14 \mathrm{mg} / \mathrm{mL}$ solution in $50 \mathrm{mM}$ sodium phosphate buffer with $150 \mathrm{mM} \mathrm{NaCl}$ at $\mathrm{pH} 6.0$ stored at $-80^{\circ} \mathrm{C}$. HSA, sodium phosphate dibasic dihydrate, sodium phosphate monobasic monohydrate, sodium chloride, and polysorbate 20 (Tween $20 \AA$ ) were purchased from Sigma-Aldrich (St. Louis, MO, USA). All of the other reagents used were of analytical grade. Prior to 
analysis, all prepared samples were filtered through a sterile Spin-X $0.22 \mu \mathrm{m}$ cellulose acetate centrifuge tube filter (Costar, Corning Incorp., Salt Lake, UT, USA) centrifuged at 8,000 rpm for two minutes.

\subsection{SEC analysis}

SEC analysis was performed using the Shimadzu LC 20 HPLC system (Shimadzu Corporation, Kyoto, Japan). PROTEIN KW-804 (7 m, $8.0 \mathrm{~mm} \times 300$ mm) (Showa Denko, Tokyo, Japan) was used as a column with the temperature set at $25^{\circ} \mathrm{C}$, while a mobile phase comprised of $50 \mathrm{mM}$ sodium phosphate buffer $\mathrm{pH} 6.0$ with $150 \mathrm{mM} \mathrm{NaCl}$ was used for the elution at a flow rate $1 \mathrm{~mL} / \mathrm{min}$. The injection volume was $60 \mu \mathrm{L}$, and the UV absorbance was measured at $278 \mathrm{~nm}$. For the dissociation and stability study of the BoNT/A, the samples were incubated at the indicated temperatures using the Eppendorf ThermoStat C (Eppendorf AG, Hamburg, Germany) while $0.01 \%$ polysorbate 20 was added to the samples to prevent the loss due to interfacial adsorption. The baseline obtained from the buffer chromatogram was subtracted from the sample's chromatogram to remove the interference of polysorbate 20 peak elution. The relative area percentage of the respective peaks were then calculated using the following formula:

$\% A=\left(A_{t} / A_{p H 6.0}^{0}\right) \times 100$

where, ' $A_{t}^{\prime}$ is the area of the respective peak at time ' $t$ ' and ' $A^{0}{ }_{p H 6.0}$ ' is the initial area of peak 1 at $\mathrm{pH} 6.0$ referred to as the undissociated state. The area percentage of aggregates was calculated with respect to the total peak areas of the same sample at the respective time.

\subsection{Molecular weight determination by MALS}

The molecular weight of the separated peaks from SEC was determined using a miniDawn TREOS light scattering system (Wyatt Technology Corp., CA, USA) equipped with a three-angled $\left(43.6^{\circ}, 90^{\circ}\right.$ and $136.4^{\circ}$ ) detector and a $685.0 \mathrm{~nm}$ laser beam. The molecular weight was processed through the software ASTRA 7 (version 7.1.3.15) and was calculated based on the $d n / d c(0.185)$ value of the BoNT/A complex.

\subsection{Neurotoxin quantification by ELISA}

The collected fraction from the SEC (diluted 500 times) was assayed for the investigation of neurotoxin content using a Botulinum Neurotoxin Type A DuoSet ${ }^{\circledR}$ ELISA kit (R\&D Systems, Inc., Minneapolis, USA, DY4489-05). Botulinum Antitoxin Equine Type A specific for botulinum neurotoxin was used as a capture antibody, and Streptavidin-HRP (streptavidin conjugated to horseradish-peroxidase) was incorporated as a detection reagent with the minimum detectable concentration at $0.39 \mathrm{ng} / \mathrm{mL}$. The plate was incubated at $25^{\circ} \mathrm{C}$ at each step. The 96 -well microplate was first coated with the plate coating buffer containing the capture antibody and incubated for 16-18 hours. Once coated, $100 \mu \mathrm{L}$ of $1 \times$ reagent diluent was dispensed into each well and incubated for two hours. Then, $100 \mu \mathrm{L}$ of standard and samples were dispensed into the designated wells. After two hours of incubation, $100 \mu \mathrm{L}$ of streptavidin-HRP was loaded into the microplate well followed by incubation at room temperature for 20 minutes. The plates were washed three times after each step with the diluted wash buffer provided in the kit. Successively, 
$100 \mu \mathrm{L}$ of color reagent was introduced into the wells. Finally, after 20 minutes, $50 \mu \mathrm{L}$ of stop solution was added into all wells to end the enzyme-substrate reaction. The absorbance was measured using a SpectraMaxM2 spectrophotometer (Molecular Devices, Sunnyvale, USA) at $450 \mathrm{~nm}$. The absorbance of the blank was subtracted from the resulting absorbance of the respective samples. The standard curve was fitted using a four-parameter logistic curve fitting method and the concentrations were determined using the equation derived from the curve fitting.

\subsection{Zeta potential and size distribution measurement by DLS}

Zeta potential measurements were carried out using a Zetasizer Nano ZS90 (Malvern Instruments, Worcestershire, UK). To avoid the corrosion of the electrodes in the cell, the sample was desalted by dialyzing $0.25 \mathrm{mg} / \mathrm{mL}$ BoNT/A complex sample against $50 \mathrm{mM}$ sodium phosphate buffer $\mathrm{pH} 4.8$ using a $0.1 \mathrm{~mL}$ volume 10,000 MWCO Slide-A-Lyzer ${ }^{\mathrm{TM}}$ mini dialysis cup (Thermo Fisher Scientific Solutions Co., Ltd., Seoul, South Korea). The dialyzed samples were then loaded into the disposable folded capillary cells (DTS1070, Malvern Instruments Ltd., Worcestershire, UK) and the zeta potential was scanned from acidic to alkaline $\mathrm{pH}$ through manual titration. $\mathrm{NaOH}$ of $1 \mathrm{~N}$ and $0.1 \mathrm{~N}$ were used to adjust the $\mathrm{pH}$. Additionally, the hydrodynamic size of $0.5 \mathrm{mg} / \mathrm{mL}$ BoNT/A complex was monitored with increasing temperature starting from $20^{\circ} \mathrm{C}$ to $80^{\circ} \mathrm{C}$ at $2^{\circ} \mathrm{C} /$ step. First, $100 \mu \mathrm{L}$ of sample was loaded into the QS High precision cell $3 \times 3 \mathrm{~mm}$ (Hellma Analytics, Müllheim, Germany) and the samples were equilibrated at the respective temperatures for 60 seconds. All the measurements were repeated three times. Zetasizer software version 7.11 was utilized to achieve the parameters from the auto-correlated function.

\section{Results And Discussion}

\section{1 pH-based dissociation of BoNT/A complex in SEC}

Figure 1a represents SEC chromatograms of the BoNT/A complex at $\mathrm{pH} 6.0, \mathrm{pH} 7.4$, and $\mathrm{pH} 8.0$ analyzed after an hour incubation at $25^{\circ} \mathrm{C}$. A dominant peak (peak 1) was observed at all three pH levels, followed by an additional peak at retention time around 10 minutes (peak 2) at pH 7.4 and 8.0. The relative area of peak 1 was reduced approximately by $5 \%$ and $8 \%$ at $\mathrm{pH} 7.4$ and 8.0, respectively. Additional analysis through MALS identified the molecular weights of peak 1 as $834.7,743.6$ and $578 \mathrm{kDa}$ at pH 6.0, 7.4 and 8.0, respectively (Figure 1a). The decrease in molecular weight along with a shift in retention times and appearance of the second peak with increasing $\mathrm{pH}$, indicated the dissociation of protein components from its complex at higher $\mathrm{pH}$ values.

The $\mathrm{pH}$ dependent assembly/disassembly of neurotoxin complexes has been confirmed previously in several research $13,14,21,36$. Furthermore, high level of stability (i.e. stability of association) between the neurotoxin and NTNHA at pH 6.0 with a dissociation constant of $\sim 30.8 \mathrm{nM}$ and no detectable interaction at $\mathrm{pH} 7.5$ observed formerly ${ }^{37}$, suggests peak 2 to be the dissociated BoNT/A owing to the basic solution $\mathrm{pH}$. However, the molecular weight of peak 2 was not detectable by MALS due to the low signal intensity. 
To verify the peak 2 as dissociated BoNT/A, a more specific analytical tool, ELISA was applied on the fractions of SEC. The DuoSet ${ }^{\circledR}$ ELISA development system used in this experiment is known to selectively detect and quantify $150 \mathrm{kDa}$ BoNT/A existing either in free or complex form. As shown in Figure 1b, a relatively larger portion of neurotoxin was detected at peak 1 at pH 6.0 indicating the greater presence of BoNT/A in an associated state. On the contrary, the ELISA result at $\mathrm{pH} 7.4$ showed the dominant presence of BoNT/A in the fraction of peak 2, signifying the dissociation of a high amount of toxin from the NAPs as it is exposed to the basic $\mathrm{pH}$. Hence, it can be established that peak 1 represents 'NAPs with undissociated BoNT/A', while peak 2 represents 'dissociated BoNT/A'.

To further investigate the concentration dependent manner of the dissociated BoNT/A from its complex, different concentrations of the BoNT/A complex were analyzed from $0.01 \mathrm{mg} / \mathrm{mL}$ to $0.5 \mathrm{mg} / \mathrm{mL}$ at $\mathrm{pH}$ 7.4 buffer (Figure 1c). To minimize the loss of dissociated BoNT/A through interfacial adsorption, polysorbate 20 was added to each solution. The area under the curve (AUC) of peak 1 was found to be linear with an $R^{2}$ value of 0.9973 . In comparison, peak 2 showed less linearity $\left(R^{2}=0.9676\right)$. The dissociation of the BoNT/A from the complex was previously found to be dependent on the complex's concentration with a high amount of dissociation at a lower concentration ${ }^{14}$, which could result in the deviation of its linearity in respect to the complex's concentration. Besides, limit of detection (LOD) and limit of quantitation (LOQ) for peak 2 were found to be $22 \mathrm{ng} / \mathrm{mL}$ and $65 \mathrm{ng} / \mathrm{mL}$ of the BoNT/A complex, respectively.

\subsection{Reversibility of the BoNT/A association}

While the current study along with earlier studies confirms the dissociation of neurotoxin at basic $\mathrm{pH}$, limited information is reported on its reversibility to the original complex once re-exposed to acidic $\mathrm{pH}$. Matsui et al. introduced the favorable formation of a complex of BoNT/A and NTNHA at acidic pH using small-angle X-ray scattering ${ }^{21}$. Sagane et al. demonstrated the reversibility of a dissociated HA subcomplex into a neurotoxin complex using SDS-PAGE ${ }^{38}$. Herein, SEC was utilized to assess the reversibility of BoNT/A with NAPs as shown in Figure 2. To evaluate its reversibility, sample solution incubated for an hour at $\mathrm{pH} 7.4$ was titrated back to $\mathrm{pH} 6.0$ using $0.6 \mathrm{M}$ hydrochloric acid. Interestingly, the SEC chromatogram of the $\mathrm{pH}$-reversed sample showed the disappearance of peak 2 compared to $\mathrm{pH}$ 7.4 (Figure 2a). Moreover, the area of peak 1 was increased, suggesting the re-association of dissociated BoNT/A into its complex form at acidic $\mathrm{pH}$. The calculated AUC-based (i.e., peak $1 ;\left[\mathrm{AUC}_{\mathrm{pH6.0}}\right] /\left[\mathrm{AUC} \mathrm{C}_{\mathrm{pH7} \text {.4 }}\right]$ ) reversibility was $81.01 \%$. To further confirm the reversibility of the complex, the collected SEC fraction was analyzed by ELISA. Supportively, the neurotoxin at peak 1 was recovered after the $\mathrm{pH}$ was adjusted back into 6.0 (Figure 2 b). The calculated BoNT/A-based reversibility was around $84.45 \%$. In summary, the propensity of the two calculated reversibility was similar and the value itself suggests high reversibility to form the initial complex. The relative loss around $16-19 \%$ could be due to the formation of insoluble/irreversible submicron or even larger particles however it was not considered further in this study. 


\subsection{Effect of time and temperature on the dissociation of BoNT/A}

The time-dependent dissociation of the BoNT/A from its complex at $\mathrm{pH} 7.4$ was investigated by incubating the sample at $25^{\circ} \mathrm{C}$ for 96 hours (Figure $3 \mathrm{a}$ and $3 \mathrm{~b}$ ). Compared to its undissociated state at pH $6.0,96 \%$ of the relative AUC of peak 1 was retained until 1.5 hours. It decreased to around $87 \%$ and $80 \%$ after 24 and 96 hours respectively with an increase in the relative AUC of peak 2 . Consequently, the dissociation occurred in a time dependent manner.

To further investigate the behavior of its dissociation and to accelerate the reaction, the incubation temperature was increased to $37^{\circ} \mathrm{C}$ in both $\mathrm{pH} 6.0$ and 7.4 for 14 days (Figure $3 \mathrm{c}$ and $3 \mathrm{~d}$ ). As a result, relatively rapid dissociation was observed at $\mathrm{pH} 7.4$ compared to the sample incubated at $25^{\circ} \mathrm{C}$. However, a new peak arose at a shorter time as the area of peak 1 and 2 decreased, indicating the formation of soluble aggregates $(<100 \mathrm{~nm})$ at both $\mathrm{pH} 6.0$ and 7.4. This demonstrates that increasing the incubation temperature from $25^{\circ} \mathrm{C}$ to $37^{\circ} \mathrm{C}$ not only accelerates the dissociation but also induces protein unfolding or partial unfolding of any subunits in the complex and even aggregation of free BoNT/A. In comparison, the aggregates increased from $14 \%$ on the 3rd day to $31 \%$ on the 14 th day at $\mathrm{pH} 7.4$, while it was only around $6 \%$ on the 14 th day at $\mathrm{pH} 6.0$, suggesting higher thermodynamic (i.e., conformational) stability at acidic $\mathrm{pH}$. On the other hand, the dissociation of BoNT/A was limited at $\mathrm{pH} 6.0$, supporting that a stronger association with NAPs increased its stability against thermal stress. Nevertheless, the result additionally demonstrates the importance of controlling the temperature for in vitro tests since it causes separate aggregation kinetics rather than just dissociation reactions.

\subsection{Isoelectric point and aggregation onset of BoNT/A}

Zeta potential and hydrodynamic properties of proteins are used as an important physical parameter to determine their colloidal stability 39,40 . To better comprehend the relationship of the surface charge properties and aggregation behavior of the BoNT/A complex with the $\mathrm{pH}$ and temperature, DLS was employed to measure its zeta potential and hydrodynamic size distribution (i.e., Z-average size).

The isoelectric point (p) of the BoNT/A complex in solution was found to be at pH 5.69 (Figure 4a), that is where net charge is close to zero. It has been suggested that the $\mathrm{p} /$ of the individual subunits within the complex should be considered to elucidate its behavior ${ }^{41}$. However, the information about the overall surface charge could be beneficial in interpreting the net stability of the complex. BoNT/A and NTNHA possess a large solvent accessible area through multivalent interfaces, which might make the interlocked interface sensitive to $\mathrm{pH}$ changes ${ }^{13}$. Considering the whole complex as a single unit, as the $\mathrm{pH}$ moves further away from the $\mathrm{p} /$ toward a basic $\mathrm{pH}$, more deprotonations occur along with an increasing number of negatively charged residues. The apparent zeta potential of the complex shifted from $-3 \mathrm{mV}$ at $\mathrm{pH} 6.0$ to approximately $-12 \mathrm{mV}$ at $\mathrm{pH}$ 7.4. As previously elucidated, the positively charged residues at $\mathrm{pH} 6.0$, particularly aspartate and glutamate present on the interface of the complex, deprotonate at $\mathrm{pH} 7.4$, generating repulsive interactions with negatively charged NTNHA and finally inducing the dissociation of 
the complex ${ }^{13,37}$. On the other hand, a high aggregation tendency was observed at pH 7.4 through SEC. Ideally, the protein-protein repulsion should be higher at $\mathrm{pH} 7.4$ since it is far from the $\mathrm{p} /$, reducing the probability of aggregation. However, in case of BoNT/A complex, the dissociation resulted in individualization of behavior of each dissociated component. Exposed free binding sites of the proteins after dissociation might have led to hydrophobic interactions forming high molecular weight species.

To confirm the aggregation behavior of BoNT/A complex at different pHs, Z-average size was analyzed with increasing temperature (Figure $4 b$ ). Prior to heating, the Z-average size of the BoNT/A complex was found to be $27.19 \mathrm{~nm}, 26.12 \mathrm{~nm}$, and $26.20 \mathrm{~nm}$ at pH 6.0, 7.4 and 8.0, respectively. Since the Z-average size provides a measure of the average particle size distribution, the initial reduction in the size as the $\mathrm{pH}$ shifts from 6.0 to 7.4 and 8.0 might specify dissociation of the neurotoxin leading to a decrease in its average size distribution. This result additionally supports the shift in the retention time detected in the SEC chromatogram toward an alkaline $\mathrm{pH}$. Upon heating, the complex at $\mathrm{pH} 7.4$ and 8.0 exhibited a similar sharp transition in the Z-average size with the aggregation onset $\left(T_{\text {onset }}\right)$ at around $52^{\circ} \mathrm{C}$. As observed for several large proteins, the temperature induced unfolding of the BoNT/A complex resulted in the formation of larger aggregates (> $1000 \mathrm{~nm}$ ). On the contrary, $\mathrm{pH} 6.0$ exhibited a slower transition curve with the $T_{\text {onset }}$ of $54^{\circ} \mathrm{C}$. Comparable to the earlier SEC result, a slower aggregation tendency was observed at $\mathrm{pH}$ 6.0. The similar thermodynamic behavior of the BoNT/A complex was also noted in earlier reports with $T_{m} \mathrm{~s}$ of $58.5^{\circ} \mathrm{C}, 56.7^{\circ} \mathrm{C}$ and $55.9^{\circ} \mathrm{C}$ at pH $6.0,7.0$, and 8.0 , respectively, using circular dichroism (CD) ${ }^{42}$. Evidently, the overlaid size distribution at $50^{\circ} \mathrm{C}$ and $54^{\circ} \mathrm{C}$ clearly depicts the state of the complex with temperatures (Figure $4 \mathrm{c}$ and $4 \mathrm{~d}$ ). These result support that $\mathrm{pH} 6.0$ is thermodynamically more stable than $\mathrm{pH} 7.4$ and that the increased stability is the result of the strong interaction between BoNT/A and NAPs. As BoNT/A dissociates from the complex, its vulnerability toward aggregation increases, which might in turn decrease the activity required for a proper therapeutic application.

\subsection{BoNT/A complex vs free BoNT/A: role of NAP in stability and future perspective}

The present work supports the earlier studies that BoNT/A within its complex at acidic pH is structurally more stable against heat stress than in a free state $24,25,42$. Most of the marketed lyophilized formulations exist in a complex form with a molecular weight ranging from $500 \mathrm{kDa}$ in Dysport ${ }^{\circledR}$ to $900 \mathrm{kDa}$ in Botox $\AA$ 16. Yet, their reconstitution $\mathrm{pH}$ value approaching the physiological $\mathrm{pH}$ increases the probability of dissociation into the free neurotoxin. It has been demonstrated that the domains of botulinum neurotoxins are highly sensitive to even mild agitation ${ }^{43}$. Therefore, the dissociation followed by aggregation of BoNT/A at neutral or alkaline $\mathrm{pH}$ could be the main reason for the necessity of human serum albumin as a stabilizer. Especially in the product Xeomin ${ }^{\circledR}$ (BoNT/A without NAPs) where twice the amount of human serum albumin in Botox® has been used. However, the risk of viral and pathogens transmission on using human serum albumin, and challenges in the analysis of the BoNT/A in their presence may require albumin-free formulations in the pharmaceutical market. Moreover, SEC results suggest that the inclusion of polysorbate 20 might still be insufficient in preventing the aggregation 
tendency due to strong hydrophobic interactions especially at elevated temperature suggesting the requirement of more efficient stabilizer. In such a scenario, formulating the BoNT/A complex products at acidic $\mathrm{pH}$ can minimize the extent of hydrophobic interactions and decrease the aggregation tendency as represented in Figure 5. In summary, the analytical tool kit (i.e., SEC, ELSA, and DLS) utilized in this study was very useful in elucidating the biophysical properties of the botulinum complex in different solutions as well as for providing a key to developing an in vitro release test method.

\section{Conclusion}

The biophysical characterization of BoNT/A complex along with its thermodynamic stability was assessed through a combination of conventional analytical tools for biopharmaceuticals. The key findings of this study are: (i) the time, temperature, and $\mathrm{pH}$-dependent dissociation of the neurotoxin from the complex, (ii) the vulnerability of the dissociated BoNT/A toward aggregation compared to its associated state within the complex, and (iii) the discovery of the reversible association of the BoNT/A with NAPs when the $\mathrm{pH}$ is titrated to acidic $\mathrm{pH}$. Overall, the data suggested that the neurotoxin is in a more stable state when it is associated with NAPs at pH 6.0 compared to its dissociated state at pH 7.4. However, it should be noted that NAPs alone cannot solely replace the use of stabilizers. Nevertheless, these insights might prove to be valuable for the future development of albumin-free BoNT/A formulations.

\section{Declarations}

\section{Competing interests}

The authors declare no conflicts of interest.

\section{Acknowledgements}

This work was supported partly by a National Research Foundation of Korea Grant funded by the Korean government (NRF-2018R1A5A2023127, NRF-2019R1A2C1083911 and NRF-2020R1I1A1A01052333)

\section{Author contributions}

S.H.: Conceptualization, Data curation, Formal analysis, Investigation, Methodology, Visualization, Writing J.C.L.: Conceptualization, Data curation, Formal analysis, Investigation, Visualization E.C.L.: Formal analysis, Investigation, Methodology S.J.: Formal analysis, Investigation, Methodology 
J.S.N.: Resources, Investigation, Methodology

B.J.Y.: Resources, Investigation, Methodology

D.H.N.: Formal analysis, Investigation, Methodology

S.H.J.: Supervision, Conceptualization, Funding acquisition, Review and Editing

N.A.K.: Supervision, Conceptualization, Data curation, Review and editing

\section{References}

1. Montecucco, C. \& Schiavo, G. Structure and function of tetanus and botulinum neurotoxins. Q. Rev. Biophys. 28, 423-472, doi:https://doi.org//10.1017/s0033583500003292 (1995).

2. Tighe, A. P. \& Schiavo, G. Botulinum neurotoxins: Mechanism of action. Toxicon 67, 87-93, doi:https://doi.org/10.1016/j.toxicon.2012.11.011 (2013).

3. Bryant, A.-M., Davis, J., Cai, S. \& Singh, B. R. Molecular Composition and Extinction Coefficient of Native Botulinum Neurotoxin Complex Produced by Clostridium botulinum Hall A Strain. Protein J. 32, 106-117, doi:https://doi.org/10.1007/s10930-013-9465-6 (2013).

4. Frevert, J. Xeomin is free from complexing proteins. Toxicon 54, 697-701, doi:https://doi.org/10.1016/j.toxicon.2009.03.010 (2009).

5. Brin, M. F. Basic and clinical aspects of BOTOX®. Toxicon 54, 676-682, doi:https://doi.org/10.1016/j.toxicon.2009.03.021 (2009).

6. Wortzman, M. S. \& Pickett, A. The Science and Manufacturing Behind Botulinum Neurotoxin Type AABO in Clinical Use. Aesthetic Surg. J. 29, S34-S42, doi:https://doi.org/10.1016/j.asj.2009.09.014 (2009).

7. Sharma, S. K. \& Singh, B. Enhancement of the Endopeptidase Activity of Purified Botulinum Neurotoxins A and E by an Isolated Component of the Native Neurotoxin Associated Proteins. Biochemistry 43, 4791-4798, doi:https://doi.org/10.1021/bi0355544 (2004).

8. Ghosal, K. J., Patel, K., Singh, B. R. \& Hale, M. L. Role of critical elements in botulinum neurotoxin complex in toxin routing across intestinal and bronchial barriers. Plos One 13, e0199524, doi:https://doi.org/10.1371/journal.pone.0199524 (2018).

9. Sakaguchi, G. Clostridium botulinum toxins. Pharmacol. Ther. 19, 165-194, doi:https://doi.org/10.1016/0163-7258(82)90061-4 (1982).

10. Lee, K. et al. Structure of a Bimodular Botulinum Neurotoxin Complex Provides Insights into Its Oral Toxicity. PLoS Pathog. 9, e1003690, doi:https://doi.org/10.1371/journal.ppat.1003690 (2013).

11. Lam, K.-H. \& Jin, R. Architecture of the botulinum neurotoxin complex: a molecular machine for protection and delivery. Curr. Opin. Struct. Biol. 31, 89-95, doi:https://doi.org/10.1016/j.sbi.2015.03.013 (2015). 
12. Sharma, S. \& Singh, B. Hemagglutinin binding mediated protection of botulinum neurotoxin from proteolysis. J. Nat. Toxins 7, 239-253, doi:https://europepmc.org/article/med/9783262 (1998).

13. Gu, S. et al. Botulinum Neurotoxin Is Shielded by NTNHA in an Interlocked Complex. Science 335, 977-981, doi:https://doi.org/10.1126/science.1214270 (2012).

14. Eisele, K.-H., Fink, K., Vey, M. \& Taylor, H. V. Studies on the dissociation of botulinum neurotoxin type A complexes. Toxicon 57, 555-565, doi:https://doi.org/10.1016/j.toxicon.2010.12.019 (2011).

15. Pirazzini, M., Rossetto, O., Eleopra, R. \& Montecucco, C. Botulinum Neurotoxins: Biology, Pharmacology, and Toxicology. Pharmacol. Rev. 69, 200-235, doi:https://doi.org/10.1124/pr.116.012658 (2017).

16. Fonfria, E. et al. The Expanding Therapeutic Utility of Botulinum Neurotoxins. Toxins 10, 208, doi:https://doi.org/10.3390/toxins10050208 (2018).

17. Chaudhuri, R., Cheng, Y., Middaugh, C. R. \& Volkin, D. B. High-Throughput Biophysical Analysis of Protein Therapeutics to Examine Interrelationships Between Aggregate Formation and Conformational Stability. AAPS J. 16, 48-64, doi:https://doi.org/10.1208/s12248-013-9539-6 (2014).

18. Maity, H., Karkaria, C. \& Davagnino, J. Effects of pH and Arginine on the Solubility and Stability of a Therapeutic Protein (Fibroblast Growth Factor 20): Relationship between Solubility and Stability. Curr. Pharm. Biotechnol. 10, 609-625, doi:https://doi.org/10.2174/138920109789069297 (2009).

19. Tollinger, M., Crowhurst, K. A., Kay, L. E. \& Forman-Kay, J. D. Site-specific contributions to the pH dependence of protein stability. Proc. Natl. Acad. Sci. 100, 4545-4550, doi:https://doi.org/10.1073/pnas.0736600100 (2003).

20. Jr., R. W. T. et al. Evaluation of effects of $\mathrm{pH}$ and ionic strength on colloidal stability of IgG solutions by PEG-induced liquid-liquid phase separation. J. Chem. Phys. 145, 185101, doi:https://doi.org/10.1063/1.4966708 (2016).

21. Matsui, T. et al. Structural Basis of the pH-Dependent Assembly of a Botulinum Neurotoxin Complex. J. Mol. Biol. 426, 3773-3782, doi:https://doi.org/10.1016/j.jmb.2014.09.009 (2014).

22. Frevert, J. \& Dressler, D. Complexing proteins in botulinum toxin type A drugs: a help or a hindrance? Biologics 4, 325-332, doi:https://doi.org/10.2147/BTT.S14902 (2010).

23. Nestor, M. S., Arnold, D. \& Fischer, D. The mechanisms of action and use of botulinum neurotoxin type A in aesthetics: Key Clinical Postulates II. J. Cosmet. Dermatol. 19, 2785, doi: https://doi.org/10.1111/jocd.13702 (2020).

24. Chellappan, G., Kumar, R., Cai, S. \& Singh, B. R. Role of Neurotoxin Associated Proteins in the Low pH Induced Structural Changes in the Botulinum Neurotoxin Complex. Protein J. 33, 557-564, doi:https://doi.org/10.1007/s10930-014-9588-4 (2014).

25. Kukreja, R. V. \& Singh, B. R. Comparative Role of Neurotoxin-Associated Proteins in the Structural Stability and Endopeptidase Activity of Botulinum Neurotoxin Complex Types A and E. Biochemistry 46, 14316-14324, doi:https://doi.org/10.1021/bi701564f (2007).

26. Kutschenko, A., Bigalke, H., Wegner, F. \& Wohlfarth, K. The role of human serum albumin and neurotoxin associated proteins in the formulation of BoNT/A products. Toxicon 168, 158-163, 
doi:https://doi.org/10.1016/j.toxicon.2019.07.005 (2019).

27. Malhotra, R., Huilgol, S. C. \& Selva, D. Botulinum Toxin and Human Serum Albumin. Arch. Ophthalmol. 121, 1661-1661, doi:https://doi.org/10.1001/archopht.121.11.1661-a (2003).

28. Kim, J. et al. Safety verification for polysorbate 20, pharmaceutical excipient for intramuscular administration, in Sprague-Dawley rats and New Zealand White rabbits. Plos One 16, e0256869, doi:https://doi.org/10.1371/journal.pone.0256869 (2021).

29. Stanker, L. H. et al. A Monoclonal Antibody Based Capture ELISA for Botulinum Neurotoxin Serotype B: Toxin Detection in Food. Toxins 5, 2212-2226, doi:https://doi.org/10.3390/toxins5112212 (2013).

30. Stanker, L. H., Merrill, P., Scotcher, M. C. \& Cheng, L. W. Development and partial characterization of high-affinity monoclonal antibodies for botulinum toxin type $A$ and their use in analysis of milk by sandwich ELISA. J. Immunol. Methods 336, 1-8, doi:https://doi.org/10.1016/j.jim.2008.03.003 (2008).

31. Keller, J. E. Characterization of New Formalin-Detoxified Botulinum Neurotoxin Toxoids. Clin. Vaccine Immunol. 15, 1374-1379, doi:https://doi.org/10.1128/CVI.00117-08 (2008).

32. Kim, Y. J. et al. Development of Capture ELISA Using a Biotinylated Monoclonal Antibody for Detection of Botulinum Neurotoxin Type A. J. Bacteriol. Virol. 38, 119-125, doi:https://doi.org/10.4167/jbv.2008.38.3.119 (2008).

33. Fekete, S., Beck, A., Veuthey, J.-L. \& Guillarme, D. Theory and practice of size exclusion chromatography for the analysis of protein aggregates. J. Pharm. Biomed. Anal. 101, 161-173, doi:https://doi.org/10.1016/j.jpba.2014.04.011 (2014).

34. Lee, J. et al. Efficacy and Safety of MT10107 (Coretox) in Poststroke Upper Limb Spasticity Treatment: A Randomized, Double-Blind, Active Drug-Controlled, Multicenter, Phase III Clinical Trial. Arch. Phys. Med. Rehabil. 101, 1485-1496, doi:https://doi.org/10.1016/j.apmr.2020.03.025 (2020).

35. Kwak, S., Kang, W.-h., Rhee, C.-H., Yang, G.-H. \& Cruz, D. J. M. Comparative Pharmacodynamics Study of 3 Different Botulinum Toxin Type A Preparations in Mice. Dermatol. Surg. 46, e132, doi:https://doi.org/10.1097/DSS.0000000000002402 (2020).

36. Chen, F., Kuziemko, G. M. \& Stevens, R. C. Biophysical Characterization of the Stability of the 150Kilodalton Botulinum Toxin, the Nontoxic Component, and the 900-Kilodalton Botulinum Toxin Complex Species. Infect. Immun. 66, 2420-2425, doi:https://doi.org/10.1128/IAl.66.6.24202425.1998 (1998).

37. Gu, S. \& Jin, R. Assembly and Function of the Botulinum Neurotoxin Progenitor Complex. Botulinum Neurotoxins, 21-44, doi:https://doi.org/10.1007/978-3-642-33570-9_2 (2012).

38. Sagane, Y. et al. Reversible Association of the Hemagglutinin Subcomplex, HA-33/HA-17 Trimer, with the Botulinum Toxin Complex. Protein J. 36, 417-424, doi:https://doi.org/10.1007/s10930-0179733-y (2017).

39. Gribenko, A. V. \& Makhatadze, G. I. Role of the Charge-Charge Interactions in Defining Stability and Halophilicity of the CspB Proteins. J. Mol. Biol. 366, 842-856, doi:https://doi.org/10.1016/j.jmb.2006.11.061 (2007). 
40. Strickler, S. S. et al. Protein Stability and Surface Electrostatics: A Charged Relationship. Biochemistry 45, 2761-2766, doi:https://doi.org/10.1021/bi0600143 (2006).

41. Leney, A. C. Subunit pl Can Influence Protein Complex Dissociation Characteristics. J. Am. Soc. Mass. Spectrom. 30, 1389-1395, doi:https://doi.org/10.1007/s13361-019-02198-3 (2019).

42. Brandau, D. T. et al. Stability of the Clostridium botulinum Type A Neurotoxin Complex: An Empirical Phase Diagram Based Approach. Mol. Pharmaceutics 4, 571-582, doi:https://doi.org/10.1021/mp0601244 (2007).

43. Toth, S. I., Smith, L. A. \& Ahmed, S. A. Extreme Sensitivity of Botulinum Neurotoxin Domains Towards Mild Agitation. J. Pharm. Sci. 98, 3302-3311, doi:https://doi.org/10.1002/jps.21676 (2009).

\section{Figures}


(a)

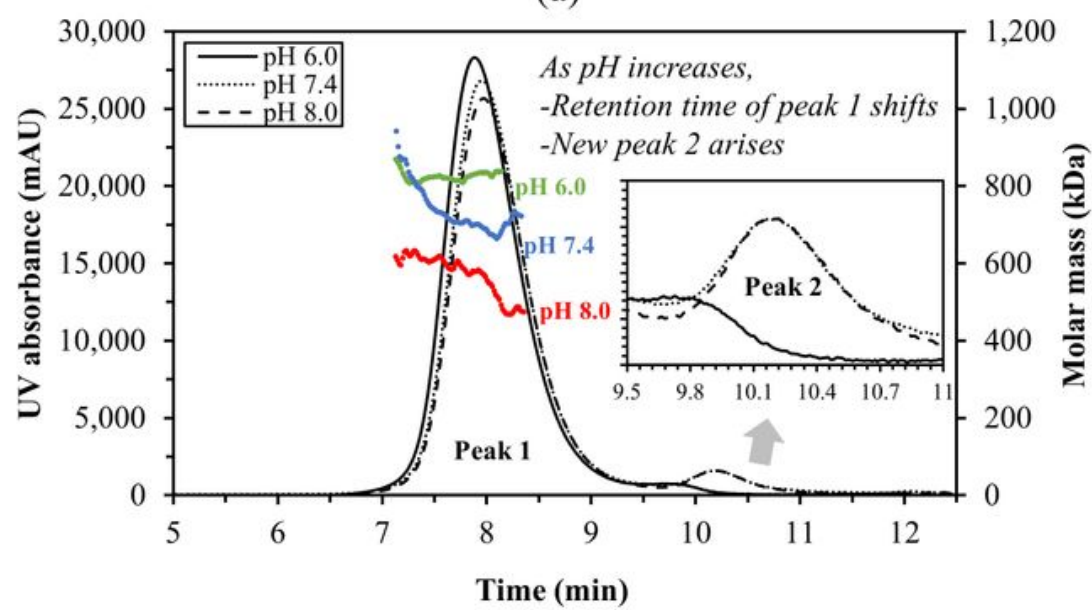

(b)

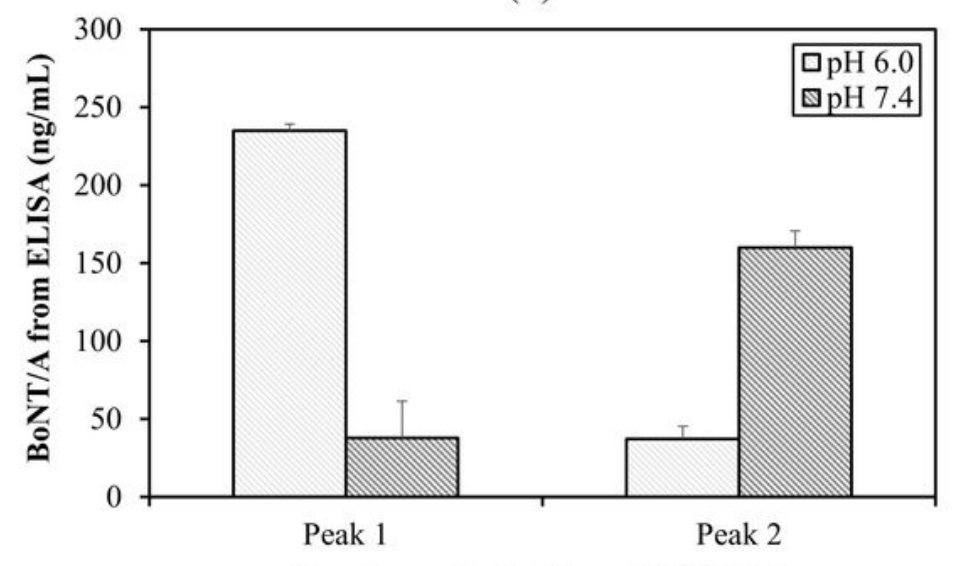

Fraction collected from SEC-HPLC

(c)

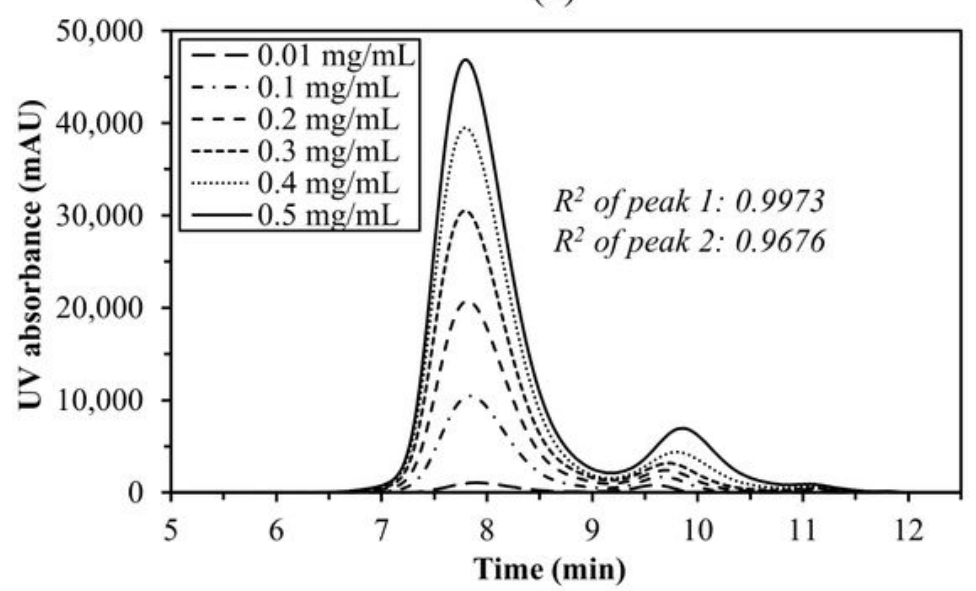

Figure 1

(a) Overlaid SEC chromatograms of $0.25 \mathrm{mg} / \mathrm{mL}$ BoNT/A complex at pH 6.0, 7.4 and 8.0 with the representation of molar mass $(\mathrm{kDa})$ obtained through MALS. (b) Concentration of BoNT/A in the collected SEC fractions from peak 1 and peak 2 at pH 6.0 and 7.4 investigated through ELISA. (c) Overlaid SEC chromatograms of BoNT/A complex at pH 7.4 in the concentration range $0.1 \mathrm{mg} / \mathrm{mL}-0.5$ $\mathrm{mg} / \mathrm{mL}$. 
(a)

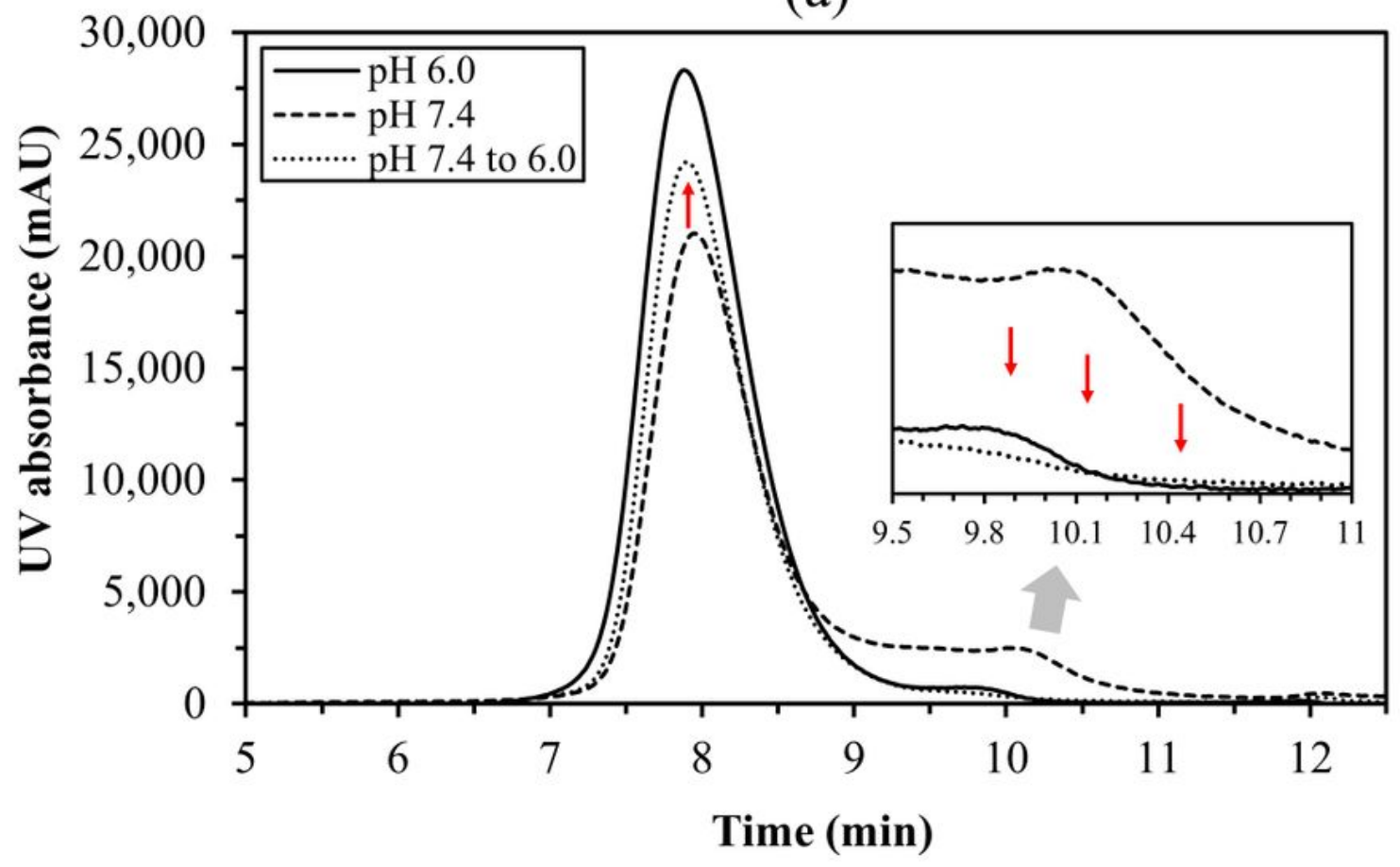

(b)

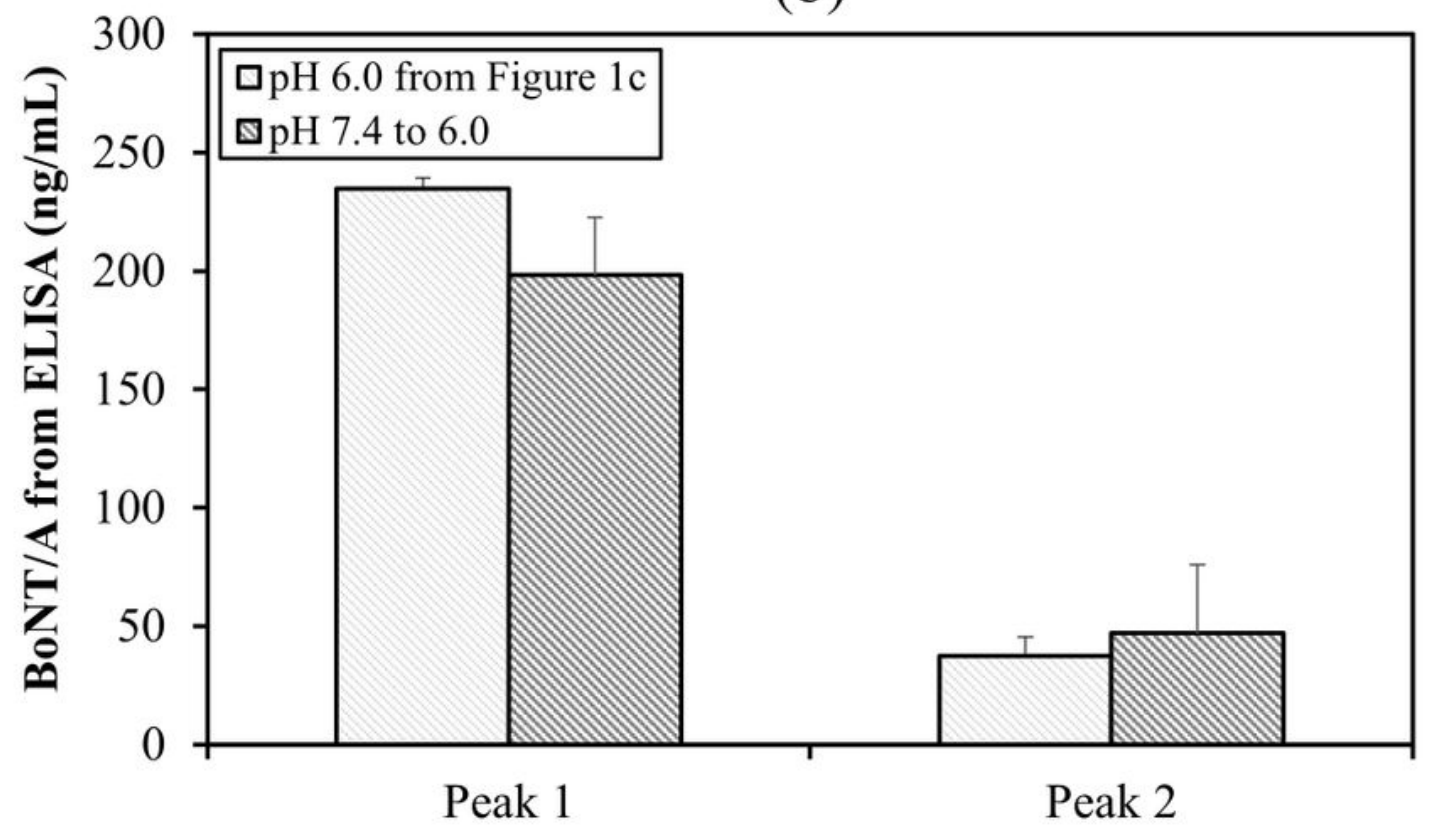

Fraction collected from SEC-HPLC

Figure 2

(a) Overlaid SEC chromatograms of $0.25 \mathrm{mg} / \mathrm{mL}$ BoNT/A complex at $\mathrm{pH} 6.0,7.4$ and $\mathrm{pH}$ titrated to $\mathrm{pH} 6.0$ after an hour incubation at $\mathrm{pH} 7.4$ and $25^{\circ} \mathrm{C}$. (b) Concentration of BoNT/A in the collected SEC fractions from peak 1 and peak 2 of the sample titrated back from pH 7.4 to $\mathrm{pH} 6.0$ investigated through ELISA. 
(a)

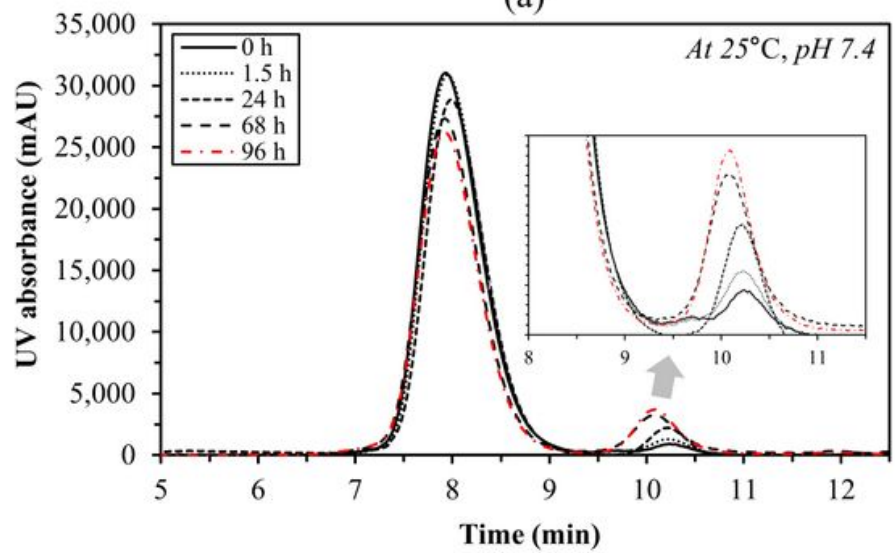

(c)

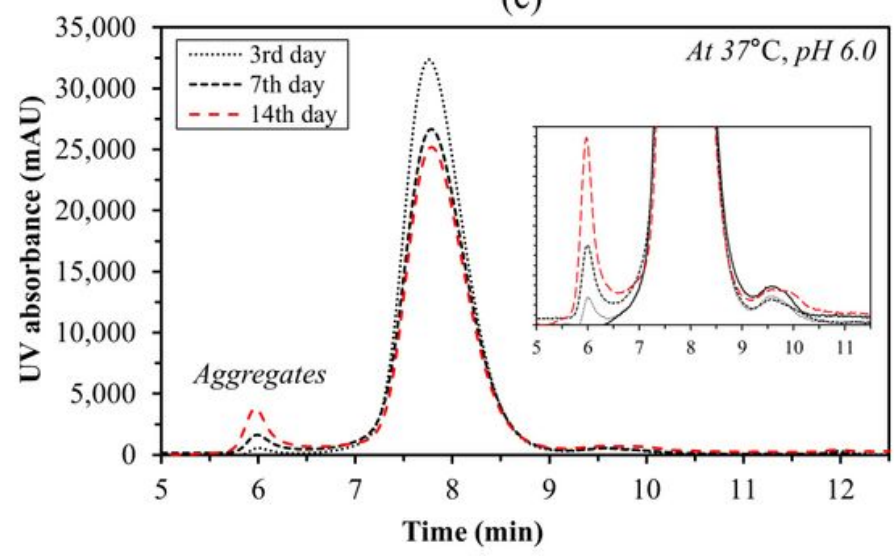

(b)

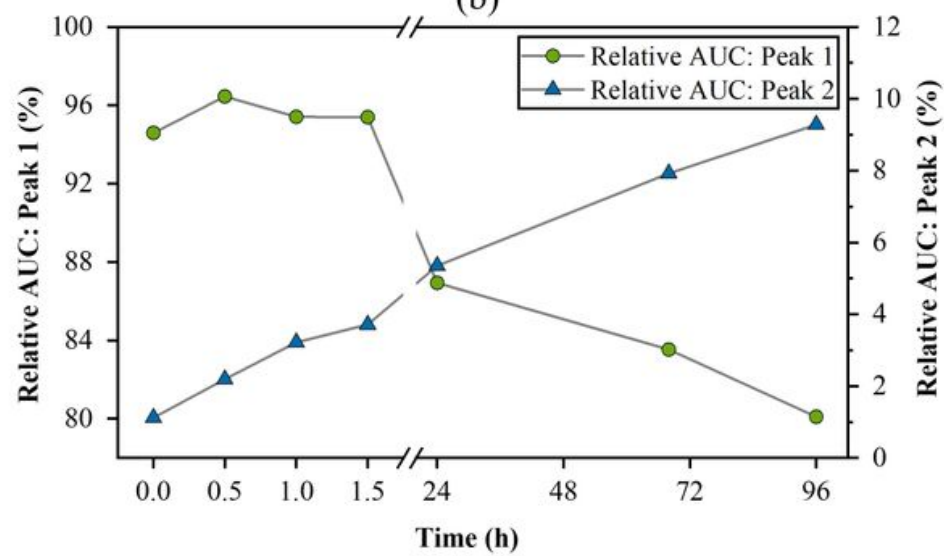

(d)

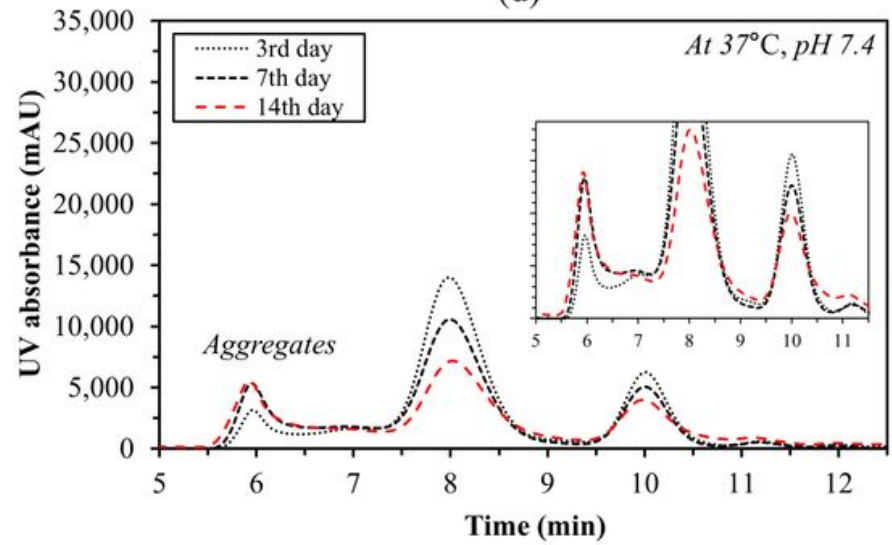

\section{Figure 3}

(a) Overlaid SEC chromatograms and (b) graph plot of BoNT/A complex at $\mathrm{pH} 7.4$ incubated at $25^{\circ} \mathrm{C}$ showing the relative area percentage of NAPs with undissociated BoNT/A and dissociated BoNT/A at different time periods. SEC chromatograms of BoNT/A complex incubated at $37^{\circ} \mathrm{C}$ in (c) $\mathrm{pH} 6.0$ and (d) pH 7.4. 
(a)

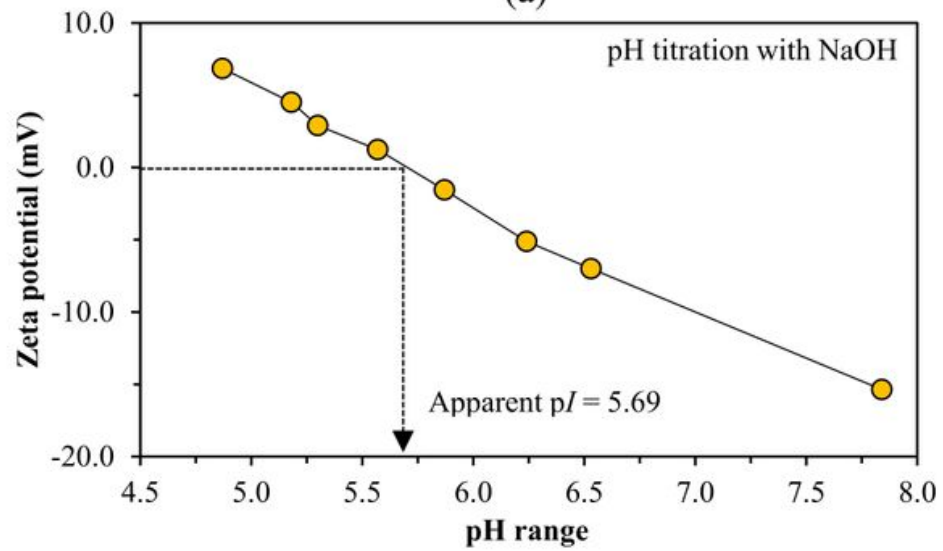

(c)

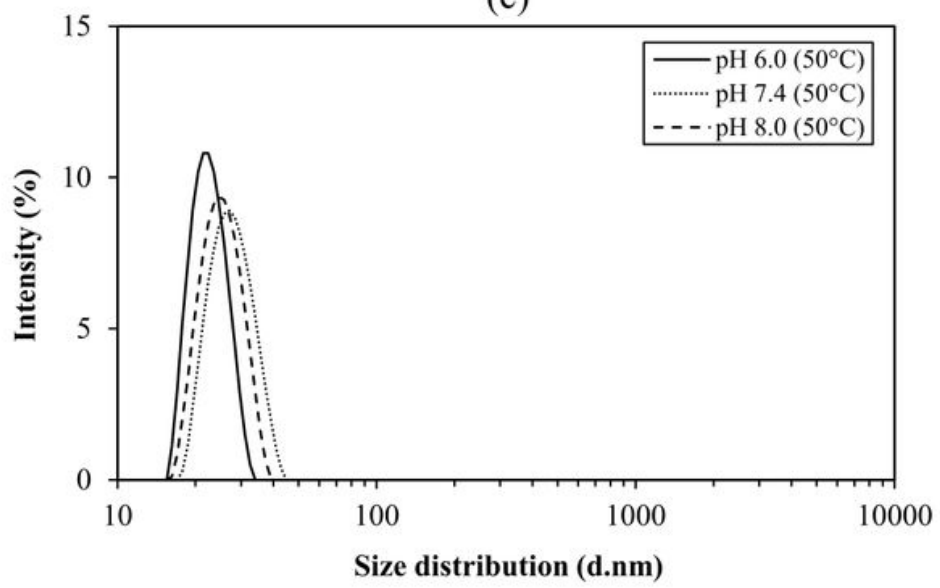

(b)

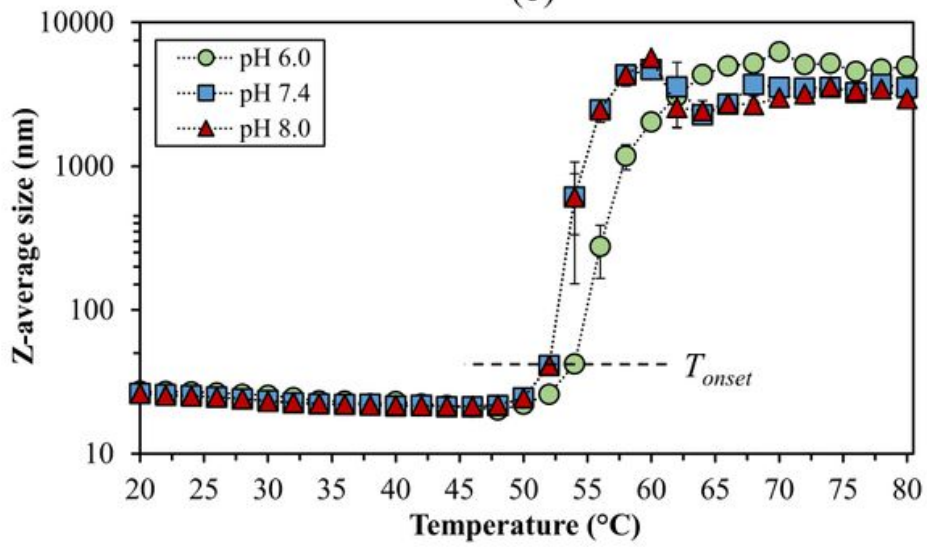

(d)

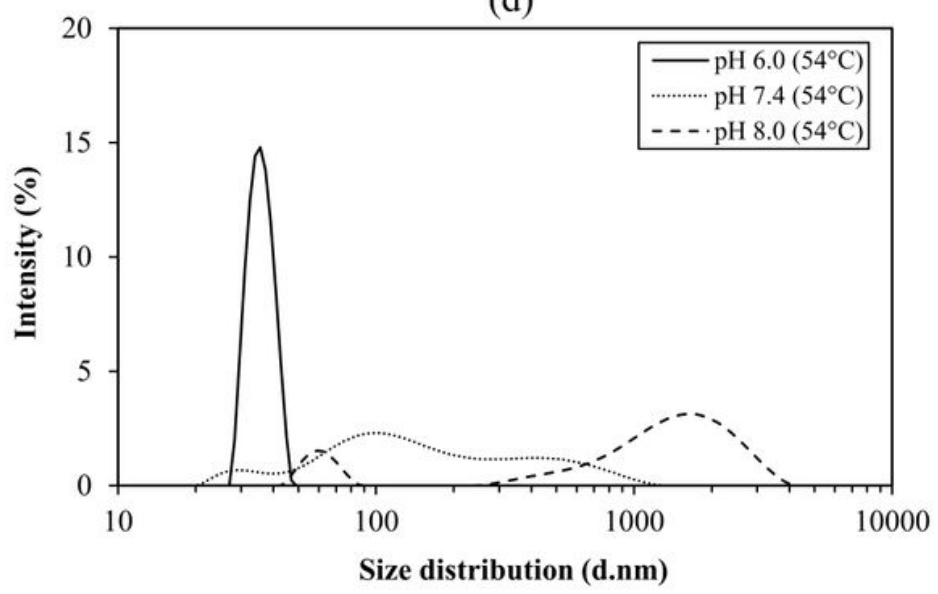

\section{Figure 4}

(a) Graph plot of zeta potential vs. pH depicting the isoelectric point of BoNT/A complex. (b) Temperature-dependent Z-average size of BoNT/A complex at different pHs. Size distribution of BoNT/A complex at temperature (c) $50^{\circ} \mathrm{C}$ and (d) $54^{\circ} \mathrm{C}$.

Associated state

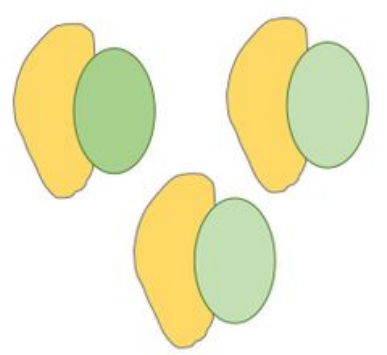

Stable at acidic $\mathrm{pH}$
Dissociated state

\section{Reversible}
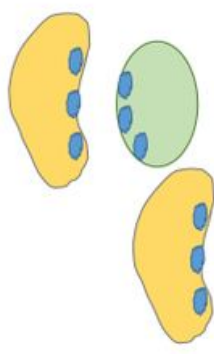

BoNT/A released at alkaline $\mathrm{pH}$

\section{Self-associated state}
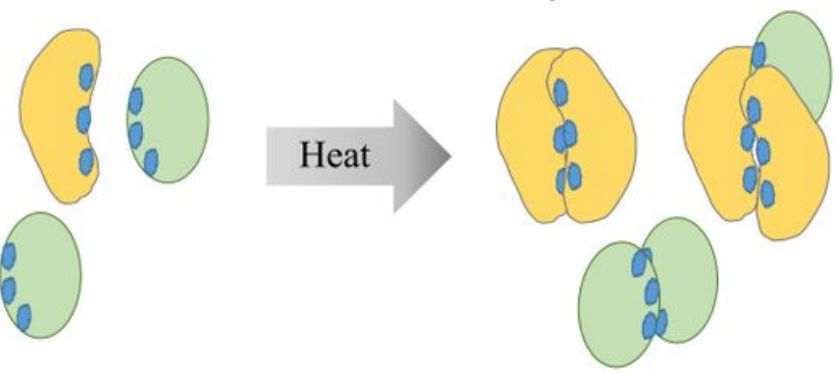

Aggregation and particulation

$$
\text { :NAPs } \bigcirc: \text { BoNT/A }
$$

\section{Figure 5}


Schematic representation of BoNT/A showing the aggregation tendency of the dissociated BoNT/A complex at alkaline $\mathrm{pH}$ 